\title{
A multicenter retrospective study of 239 patients aged over 70 years with diffuse large B-cell lymphoma in China
}

\section{Chunli Yang}

West China Hospital of Sichuan University

Qiaoer Li

West China Hospital of Sichuan University

Li Li

Sichuan Cancer Hospital

Ke Xie

Sichuan Academy of Medical Sciences \& Sichuan Provincial People's Hospital

Yakun Zhang

Affiliated Hospital of North Sichuan Medical College

Yunwei Han

Affiliated Hospital of Southwest Medical University

Liqun Zou ( $\nabla$ hxlcyxy@163.com )

West China Hospital of Sichuan University

\section{Research Article}

Keywords:

Posted Date: February 7th, 2022

DOI: https://doi.org/10.21203/rs.3.rs-1257590/v1

License: (9) This work is licensed under a Creative Commons Attribution 4.0 International License. Read Full License 


\section{Abstract}

\section{Background}

Diffuse large B-cell lymphoma (DLBCL) is the most common aggressive lymphoma subtype worldwide and frequently occurs in the elderly population. However, there was a lack of large series of data regarding the clinical profiles of over 70 years in DLBCL patients in China. We aim to summarize this population's clinical features and survival outcomes in the real world.

\section{Methods}

The multicenter retrospective study was conducted in Western China from Jan 2012 to July 2020 to investigate clinical features and survival outcomes of 239 DLBCL patients over 70 years. All patients underwent pre-treatment evaluations, treatment, and follow-up at local hospitals. The primary endpoints of this study were progression-free survival (PFS) rate and overall survival (OS) rate at two years. The second endpoints included median PFS and OS, 5-year PFS and OS rates, and adverse events during treatment.

\section{Results}

With a median follow-up of 50 months (range, 1-102), the 2-year PFS and OS rates were $53.0 \%(95 \% \mathrm{Cl}$ : 46.5-59.5) and 65.5\% (95\% Cl: 59.4-71.2). The median PFS and OS were 42.1 months and 96.4 months, and the 5-year PFS and OS rates were $44.7 \%$ (95\% Cl: 38.0-51.4) and 56.1\% (95\% Cl: 49.4-62.8). Hematological toxicities were the most common adverse effects in this study, accounting for $90.4 \%$, and leukopenia was the most frequently observed $\geq$ grade 3 events. Moreover, we observed that no rituximabcontaining treatment and chemotherapy cycles $<6$ were significantly associated with inferior survival. Furthermore, in the younger than 80 years group, reduced chemotherapy dose was associated with remarked shorter OS; the 2-y OS rate was $74.4 \%(95 \% \mathrm{Cl}$ : $67.0-81.8)$ in full dose group in comparison with $67.1 \%$ (95\% Cl: 51.8-82.4) in decreased dose group, $p 0.044$.

\section{Conclusions}

Our study indicated that rituximab, enough treatment cycles, full chemotherapy dose should be administered for elderly DLBCL patients under the balance of benefit and risk.

\section{Introduction}

Diffuse large B-cell lymphoma (DLBCL) is the most common aggressive lymphoma subtype worldwide, accounting for $30 \sim 40 \%$ of non-Hodgkin lymphoma ${ }^{[1]}$. From the SEER (Surveillance, Epidemiology, and End Results Program) database, the incidence of DLBCL increases with age; the median diagnosis age was 66 years $^{[2]}$. Compared to DLBCL patients younger than 60-70 years, older patients had a worse prognosis $^{[3-5]}$. Moreover, most clinical trials excluded these patients, leading the optimal treatment options and the survival outcome in the real-world remain to be elucidated, especially for over 70 years. 
R-CHOP is the standard regimen for the first-line DLBCL treatment ${ }^{[6,7]}$, reaching a $60 \%$ cure rate for the entire cohort ${ }^{[8]}$. Whereas most elderly patients had poor physical state and were frequently accompanied by other diseases, which limited the usage of standard dose of the regimen and further had a worse impact on the survival. Thus, dose reduce regimens or no-anthracycline-containing treatment were explored in clinical trials, such as R-miniCHOP, R-GemOx, R2 ${ }^{[9-11]}$, which resulted in a balance of efficacy and tolerance in selected elderly DLBCL patients. In the United States, the 5-year survival rate was 78.4\% in the DLBCL population of those younger than 55 years, while $54.3 \%$ was for people older than 65 years $^{[2]}$. In China, it reported that 5 -year OS rate $51.9 \%$ for DLBCL patients aged over 60 years between 2006 to 2012 in Beijing of 349 cases, and $42.8 \%$ for aged over 70 years of 145 patients $^{[12]}$. To date, there has been a limited number of studies regarding the clinical profiles of aged over 70 years in DLBCL patients. Furthermore, as the anti-CD20 antibody was widely applied in clinical practice in Western China, and better administration of treatment related adverse effects in recent years than ever, indicating that the survival outcome of Chinese elderly DLBCL needs to be investigated.

In this study, we collected 239 DLBCL patients aged over 70 years from five university hospitals in Western China to summarize the clinical features, analyze survival outcomes in the real world, and examine the factors that influenced the prognosis.

\section{Methods}

We retrospectively reviewed the record of all newly diagnosed DLBCL patients who were aged over 70 years at five hospitals located in southwest China from Jan 2012 to July 2020, including West China Hospital, Sichuan University; Sichuan Cancer Hospital \& Institute, Sichuan Provincial People's Hospital; Affiliated Hospital of North Sichuan Medical College and Affiliated Hospitals of Southwest Medical University. In all cases, the pathology diagnosis was confirmed by expert hematopathologists at these five hospitals per the World Heathy Organization classification of hematopoietic and lymphoid tumors ${ }^{[13]}$. Critical exclusion criteria were age less than 70 , central nervous system lymphoma involvement at diagnosis.

All patients underwent pre-treatment evaluations, treatment, and follow-up at the local hospital mentioned above. Evaluations included patient demographics, blood test, serum LDH, bone marrow detection. The staging was determined per the Ann Arbor staging system, and the treatment efficacy was evaluated by the enhanced computed tomography scans (CT) or positron emission tomography scans (PET-CT). Han's algorithm was applied to divide patients into germinal center B-cell (GCB) and non-GCB subtypes. The bulky disease was defined as any mass diameter exceeding $7 \mathrm{~cm}$. The investigators also reviewed the therapy options, regimens, cycles, and adverse events. Reduced dose chemotherapy in this study was explained as a decrease of $20 \%-50 \%$ of standard dose.

The primary endpoints of this study were PFS rate and OS rate at two years. The second endpoints included median PFS and OS, 5-year PFS and OS rate, adverse events during treatment. The PFS was calculated from the diagnosis to disease progression, recurrence, or any cause of death, and the OS was 
from the diagnosis to death for any reason or last follow-up. The study was done under regulatory requirements and approved by the Ethics Committee of West China Hospital of Sichuan University.

The statistical methods of comparing the difference between the groups were the $\chi 2$ test. We summarized PFS and OS using the Kaplan-Meier method, compared the difference between groups on PFS and OS with the log-rank test. Univariate and multivariate analyses related to survival were through the Kaplan-Meier method, the Cox proportional hazard models and the log-rank test. All data were analyzed with SPSS 25.0 (IBM Corp., Armonk, NY), and a two-sided $p<0.05$ was considered a statistically significant difference.

\section{Results}

\section{Patient characteristics}

We identified 316 patients newly diagnosed with DLBCL aged over 70 years from Jan 2012 to July 2020 . Of these screened patients, 22 cases had other malignant tumors concurrent, and 55 patients had only pathological diagnosis were excluded; 239 patients were finally included in the analyses.

Clinical characteristics are listed in Table1. The median age at diagnosis was 75.4 years (range, 70-91), and the ratio of male to female was 1.5:1. Staged III/IV patients were accounted for $59.8 \%$, and $27.2 \%$ for extranodal involved more than one site. There were 200 patients had subtypes via Han's algorithm, $21.8 \%$ for GCB, $61.9 \%$ for non-GCB, and $16.3 \%$ of patients remained unknown. According to the IPI score, $25.5 \%$ of cases were evaluated as a high-risk group. It was $85.8 \%$ of patients accompanied by other diseases. From the subgroups analysis data, between patients younger and older than 80 years, we observed no significant difference at baseline on clinical features.

\section{Treatment and response}

As presented in Table2, 239 cases were received initial treatment, including $64.4 \%$ for chemotherapy, two instances for radiotherapy, four for surgery, and $28.5 \%$ for combinatory therapy options. Overall, there were 219 patients administered with chemotherapy alone or with rituximab (Table3), 1029 treatment cycles were achieved, and the median cycles for a patient were 5 (range, 1-11). The most common chemotherapy regimen applied in this study was $\mathrm{CHOP}$, accounting for $84.5 \%$, other regimens comprising CVP, BR or oral vp16, and CTX. Due to the heavy financial burden of the family before 2017, when the price decreased, $34.7 \%$ of patients were treated without rituximab in this cohort. It was similar between the age under 80 years and over 80 years on the usage of rituximab; however, in the age over 80 years group, dose reduction was occurred in more patients than those aged $70-79$ years ( $45.1 \%$ vs. $20.1 \%$, $p<0.001)$.

\section{Survival outcomes}

With a median follow-up of 50 months (range, 1 - 102), 101 cases died during the treatment and followup; the median PFS and OS were 42.1 months and 96.4 months in total (Figure1). The 2-year PFS and OS 
rate of the entire cohort were 53.0\% (95\% Cl: 46.5-59.5) and 65.5\% (95\% Cl: 59.4-71.2) (Figure1). The 5year PFS and OS were 44.7\% (95\% Cl: 38.0-51.4) and 56.1\% (95\% Cl: 49.4-62.8) (Figure1). Between patients younger and older than 80 years, the 2-y PFS and OS rate were showed no remarked difference (Supplementary figure1), which were $54.5 \%$ (95\% Cl: 47.4-61.6) and $68.0 \%$ (95\% Cl: 61.5-74.5) in the younger group, compared with $45.3 \%$ (95\% Cl: 29.4-61.2) and $52.9 \%(95 \% \mathrm{Cl}: 37.0-68.8)$ in the older group.

The impact of rituximab on PFS and OS was also evaluated in our study. We found rituximab involved therapy could pronounced improve the OS and PFS for the entire cohort, the 2-y OS rate was $72.9 \%$ (95\% Cl: $65.9-80.1)$ in the rituximab group and $56.8 \%(95 \% \mathrm{Cl}: 44.5-69.2)$ in the no-rituximab group, and 5-y OS rate was $63.6 \%$ (95\% Cl: 55.2 - 72.0) vs. 50.1\% (37.6-62.6) (Figure2), p 0.008. The 2-y PFS rate was 59.5\% (95\% Cl: 51.7-67.3) in rituximab-containing treatment group and $44.2 \%$ (95\% Cl: 31.9 - 56.5), and 5-y PFS rate was $50.5 \%$ (95\% Cl: $42.1-58.9)$ vs $40.7 \%$ (28.4-53.0) (Figure2), $p 0.031$. In addition, stratified by age, patients older than 80 could benefit from rituximab both on PFS and OS. In comparison, for younger than 80 years patients, we observed the improvement in PFS and OS from rituximab, but the $p$ values were not significant (Supplementary figure2).

Reduced chemotherapy dose of regimens was frequently delivered in clinical practice for elderly lymphoma patients; in this study, we detected its role on survival outcomes. As shown in Figure3, there was no statistical difference between standard dose and reduced dose groups overall; however, in the younger than 80 years group, reduced dose was associated with remarked shorter OS, the 2-y OS was $74.4 \%$ (95\% Cl: 67.0-81.8) in full dose group in comparison with 67.1\% (95\% Cl: 51.8-82.4) in decreased dose group, $p$ 0.044. Meanwhile, we did not observe the remarked deterioration on PFS or OS in the older than 80 years group with dose reduction (Supplementary figure3).

Additionally, we observed that treatment cycles (chemotherapy \pm rituximab) were associated with survival outcomes. As Figure4 presented, patients treated with six cycles or more obtained a superior OS and PFS than that with less than six cycles, the 2-y PFS were $66.2 \%$ vs. $44.5 \%, p 0.000$, and 2-y OS were $77.6 \%$ vs. $59.5 \%, p 0.000$. Further subgroup analysis showed that $\geq 6$ cycles of R-CHOP/CVP treatment were achieved better PFS and OS than less than six cycles in the cohort, $68.7 \%$ and $49.0 \%$ for $2-y$ PFS, $p 0.001$, $81.0 \%$, and $63.8 \%$ for 2-y OS, $p 0.000$ (Supplementary figure4).

IPI score was frequently applied to predict the prognosis of DLBCL patients. Here, we used IPI score to divide patients into IPI $\leq 3$ or $I \mathrm{PI}>$ three groups; as presented in the Figure5, patients with $\mathrm{IPI} \leq 3$ had longer PFS and OS than those with IPI > 3, the 2-y PFS were $58.7 \%$ vs. 35.7\%, $p 0.005$; and 2-y OS were $71.5 \%$ vs. $47.3 \%, p 0.001$, individually. In younger than 80 years population, the 2-y PFS rate in the group of $\mathrm{IPI} \leq 3$ and of $\mathrm{IPI}>3$ was $61.2 \%, 31.8 \%, \mathrm{p} 0.001$, the $2-\mathrm{y}$ OS rate in the group of $\mathrm{IPI} \leq 3$ and of $\mathrm{IPI}>3$ was $74.9 \%, 44.7 \%, \mathrm{p} 0.000$. In $\geq 80$ years population, the 2 -y PFS rate in group of IPI $\leq 3$ and of IPI $>3$ was $43.6 \%, 49.0 \%, p 0.741$, the 2 -y OS rate in group of IPI $\leq 3$ and of IPI $>3$ was $51.7 \%, 56.3 \%, p 0.888$ (Supplementary figure5). However, further analysis in patients with the IPI $=1,2,3,4$, 5, we only found the 
OS difference on the IPI = three $v s . \mathrm{IPI}=4$ and IPI =4 vs. IPI =5 groups (Supplementary figure6 and supplementary table1), yet PFS benefit was negative.

In addition, we performed the univariate and multivariate analyses in this study. From the univariate analyses results (Supplementary table2), ECOG score $\geq 2$ ( $p$ 0.015), Ann Arbor stage III/IV ( $p$ 0.012), IPI score $>3(p 0.005)$, treated without rituximab ( $p 0.002)$ were associated with poor PFS; while extra nodal involved more than 1 site ( $p$ 0.039), ECOG score $\geq 2$ ( $p 0.002)$, IPI score $>3(p 0.001)$ and treated without rituximab ( $p$ 0.000) were linked with poor OS. As to the impact of GCB and non-GCB subtype on survival, we found no statistical significance on PFS and OS. In multivariate analyses, Ann Arbor stage III/IV was related to the poor PFS, HR 1.640 (95\% Cl: 1.031-2.610, $p$ 0.037); dose reduction was associated with the poor OS, HR 1.825 (95\% Cl: 1.056-3.154, $p 0.031$ ), and rituximab usage was related with OS benefit, HR 0.455 (95\% Cl: $0.264-0.785, p 0.005)$ (Table 4A and 4B).

\section{Toxicity}

The data of 187 patients were available to perform safety analysis; the most common adverse effects in this study were hematological toxicities, which accounted for $90.4 \%$, and leukopenia (< lower limit of normal) was the most frequently happened of $\geq$ grade 3 events (Table 5). The severity of treatmentemergent adverse events was similar between groups (Table 6). A higher incidence of fatigue, hypoalbumin were reported in older group, $63.9 \%$ vs. $93.1 \%$; $53.8 \%$ vs. $89.7 \%$. Whereas thrombocytopenia (any grade) was more common in the younger group, $34.8 \%$ vs. $13.8 \%$.

\section{Discussion}

To our knowledge, this is the largest series that has analyzed clinical features and survival outcomes in aged $\geq 70$ years DLBCL patients in China. The prognosis of this elderly DLBCL population with a 2-y OS rate of $65.5 \%$ was better than that reported in China with $42.8 \%{ }^{[12]}$. Considering two significant reasons that may contribute to the disparity, one was a higher proportion of rituximab usage in our study, $65.3 \%$ vs. $53.8 \%$, which significantly impacted the overall survival for this population, including the very elderly DLBCL patients ( $\geq 80$ years). The other was that a larger series of 239 elderly DLBCL patients aged $\geq 70$ years in our cohort, whereas 145 cases were included in that study, which may lead to the results bias.

Rituximab could improve the EFS and OS for 60-80 years DLBCL patients ${ }^{[14,15]}$. In this cohort, we found similar results: rituximab increased 2-y OS rate against only CHOP, 72.9\% vs. 56.8\%, $p 0.008$; and 2-y PFS $59.5 \%$ vs. $44.2 \%, p 0.031$. Further analysis showed this result could continue to be made in the $\geq 80$ years subgroup. In the younger than 80 years group, we could see the improvement on the PFS and OS, but the $p$ values were not significant, which may be related to the small sample size. Currently, rituximab is delivered with $375 \mathrm{mg} / \mathrm{m}^{2}$ with an interval of every three weeks for 6-8 cycles in the standard regimen; additionally, some studies investigated whether increased dose of rituximab could improve treatment efficacy in elderly patients, showing that $500 \mathrm{mg} / \mathrm{m}^{2}$ rituximab enhanced the PFS and OS of elderly male cases compared to the normal dose ${ }^{[16-18]}$. Meanwhile, a study from Netherland presented that rituximab 
intensification during the first four cycles of R-CHOP14 had no improvement in the complete response rate, 3-y PFS rate, and 3-y OS rate compared with standard R-CHOP14 and increased the toxicities in the patients between ages $66-80$ years $^{[19]}$. There were no patients administered with this maximum rituximab dose in this study. In clinical practice, it is recommended that eight cycles of R plus six cycles of CHOP21 or six cycles R-miniCHOP in treating elderly DLBCL according to some critical studies results ${ }^{[15,20-23]}$. The median cycles applied with a patient in our cohort were 5 , of which $51.5 \%$ were administered with less than six cycles of treatment, while $48.5 \%$ with six cycles or more. In contrast to usage with $\geq 6$ cycles of therapy in our data, patients with less than six cycles had inferior PFS and OS, suggesting the 6 or 8 cycles of treatment is emphasized in clinical practice.

The treatment strategy for elderly DLBCL patients has not been well established. In comparison with RCHOP14 applied in the 60-80-years old DLBCL patients, R-CHOP21 could attain similar efficacy and decreased frequency and severity of hematological adverse effects and red-blood-cell transfusion, leading the R-CHOP21 as the frequently administered regimen ${ }^{[21,24]}$. In our study, $74.4 \%$ of patients were administered with full-dose for those aged $70-79$ years and $67.1 \%$ for those older than 80 years. RminiCHOP is recommended in $\geq 80 y$ cases $^{[7,9,25]}$. Peyrade $F$ reported that $\geq 80 y$ European patients treated with R-miniCHOP obtained a 2-y OS rate of 59\%, 2-y PFS rate $47 \%{ }^{[9]}$. From our data, we observed similar results in the Chinese population with a 2-y OS rate of $52.9 \%$ and a $2-y$ PFS rate of $45.3 \%$ treated with full dose or reduced dose therapy. Additionally, in our subgroup analysis, we found that reduced chemotherapy dose was not correlated with the 2-y OS rate compared with the standard dose in older than 80 years population, which was comparable to results of some studies ever reported ${ }^{[9,25]}$, indicating that reduced chemotherapy dose may balance the efficacy and side effects for this population. However, in patients aged $70-79$ years, chemotherapy dose reduction $(\geq 20 \%)$ could have a worse impact on the OS than those with the full dose, 2-y OS rate $67.1 \%$ vs. $74.4 \%, p 0.044$. In line with a previous study result ${ }^{[26]}$, it reported that $70 \%$ of standard dose would affect the OS and EFS in patients aged over 70 years. Taken together, our data suggested that reduced chemotherapy dose exerted survival deterioration for all aged $\geq 70$ years patients, except for over 80 years.

DLBCL is divided into GCB, $A B C$ and unclassified subtypes with a gene expressing profile (GEP) ${ }^{[27]}$. It also reported that Han's algorithm had prognosis value with the cell of origin (COO) determination ${ }^{[28]}$. In our study, we applied the Han's algorithm to divide patients into GCB and non-GCB subtypes. Additionally, Mareschal $S$ et al. reported that $A B C$ subgroup was more common in elderly patients ${ }^{[29]}$. In agreement, a similar result was observed in our study with $21.8 \%$ of GCB subtype and $61.9 \%$ of non-GCB subtype. Lenz reported that GCB subtype has superior PFS and OS rates than $A B C$ subtype with $\mathrm{R}-\mathrm{CHOP}^{[30]}$. Our cohort found that GCB subtype with 2-y PFS rate $63.5 \%$ and 2-y OS rate $72.2 \%$, whereas non-GCB subtype with 2y PFS rate $55.8 \%$ and $2-y$ OS rate $66.5 \%$ (data not shown); however, the difference was not significant. Moreover, in the univariate analyses, patients with GCB or non-GCB had similar OS and PFS, associated with the non-GCB dominance in this cohort. 
IPI score is a prognostic model widely performed in DLBCL patients. Recently, a study reported that NCCN-IPI had the greatest absolute difference in OS estimates between the highest- and lowest-risk groups and best-discriminated OS compared to the IPI score and the aaIPI for the entire DLBCL cohort $^{[31]}$. In our study, NCCN-IPI and aaIPI were not fit for evaluating the prognosis as the included patients were all aged $\geq 70$ years. According to the IPI score, there were significant discriminations in PFS and OS between the IPI $\leq 3$ and IPI $>3$ groups, which remained in the subgroups analysis of younger or older than 80 years patients, suggesting that IPI score could distinguish the prognosis in elderly patients with a highrisk group or not. Moreover, we observed inferior survival outcomes in cases with IPI=5 than with IPI=4. However, it presented no discriminated PFS and OS in low-risk, low-intermediate, high-intermediate, indicating a prognostic model to evaluate in elderly DLBCL patients is an urgent need. Recently, a study from Italy reported that combined the comprehensive geriatric assessment (CGA) with IPI score could identify three risk groups with the remarked difference in terms of $\mathrm{OS}^{[32,33]}{ }^{3}$; however, we did not perform this method on our cohort as the CGA lack.

Nonetheless, this study had several limitations. First, our study was retrospective, selection and information bias could not have been avoided. Second, in our research, $34.7 \%$ of patients were treated without rituximab, due to the heavy financial burden of the family before 2017 , which may exert a deteriorating impact on the whole cohort survival. Third, patients in our study had not done the CGA, which could predict OS and may assist clinicians in determining the treatment with full-dose chemotherapy or not ${ }^{[34,35]}$.

\section{Conclusion}

This study presented that rituximab improved the PFS and OS for the older than 70 years patients with DLBCL. Additionally, reduced chemotherapy dose had a deteriorated influence on the OS for patients 7079 years; however, it did not affect the survival outcome of older than 80 years. Furthermore, less than six cycles were related to the inferior OS and PFS. Collectively, rituximab, enough treatment cycles, full chemotherapy dose, and more efficient regimens with lower toxicities should be administered for elderly DLBCL patients under the balance of benefit and risk. To this end, a simplified predictive model to optimize the stratified therapy needs to be explored in the future.

\section{Abbreviations}

\section{DLBCL}

Diffuse large B-cell lymphoma

PFS

Progression-free survival

os

Overall survival

EFS

Event-free survival 
GCB

Germinal center B-cell

GEP

Gene expressing profile

COO

Cell of origin

CT

Computed tomography scans

PET-CT

Positron emission tomography scans-computed tomography

CGA

Comprehensive geriatric assessment

IPI

International prognostic index

aalPI

age-adjusted IPI

NCCN-IPI

National Comprehensive Cancer Network prognostic index

\section{Declarations}

\section{Ethics approval and consent to participate}

The protocol of this retrospective study, involving individuals' clinical data collection, was approved and the need for informed consent was waived by the Ethics Committee of West China Hospital of Sichuan University.

\section{Consent for publication}

Not applicable

\section{Availability of data and material (ADM)}

All data generated or analyzed during the present study are included in this article. The authors declare that materials described in the manuscript, including all relevant raw data, will be freely available to any scientist wishing to use them for non-commercial purposes, without breaching participant confidentiality. The authors confirmed that all methods were performed in accordance with the relevant guidelines and regulations

\section{Competing interests}

The authors declare no competing interests. 


\section{Funding}

Not applicable.

\section{Authors' contribution}

YC and LQ contributed equally to this work. ZL: conception and design. $L L, X K, Z Y$, and HY: administrative support. YC and LQ collected and analyzed data, prepared all the figures and tables. All authors wrote and reviewed the manuscript.

\section{Acknowledgement}

The authors thank all those who participated in patient recruitment and helped gather patients' information.

\section{References}

1. Al-Hamadani M, Habermann TM, Cerhan JR et al. Non-Hodgkin lymphoma subtype distribution, geodemographic patterns, and survival in the US: A longitudinal analysis of the National Cancer Data Base from 1998 to 2011. Am J Hematol 2015; 90: 790-795.

2. https://seer.cancer.gov/statfacts/html/dlbcl.html.

3. A predictive model for aggressive non-Hodgkin's lymphoma. The New England journal of medicine 1993; 329: 987-994.

4. Zhou Z, Sehn LH, Rademaker AW et al. An enhanced International Prognostic Index (NCCN-IPI) for patients with diffuse large B-cell lymphoma treated in the rituximab era. Blood 2014; 123: 837-842.

5. Choi JH, Kim TM, Kim HJ et al. Multicenter Retrospective Analysis of Clinical Characteristics, Treatment Patterns, and Outcomes in Very Elderly Patients with Diffuse Large B-Cell Lymphoma: The Korean Cancer Study Group LY16-01. Cancer Res Treat 2018; 50: 590-598.

6. Tilly H, Gomes da Silva M, Vitolo U et al. Diffuse large B-cell lymphoma (DLBCL): ESMO Clinical Practice Guidelines for diagnosis, treatment and follow-up. Annals of oncology : official journal of the European Society for Medical Oncology 2015; 26 Suppl 5: v116-v125.

7. https://www.nccn.org/guidelines/guidelines-detail?category=1\&id=1480.

8. Nowakowski GS, Chiappella A, Witzig TE et al. ROBUST: Lenalidomide-R-CHOP versus placebo-R$\mathrm{CHOP}$ in previously untreated $\mathrm{ABC}$-type diffuse large B-cell lymphoma. Future oncology (London, England) 2016; 12: 1553-1563.

9. Peyrade $F$, Jardin F, Thieblemont $C$ et al. Attenuated immunochemotherapy regimen (R-miniCHOP) in elderly patients older than 80 years with diffuse large B-cell lymphoma: a multicentre, single-arm, phase 2 trial. The Lancet Oncology 2011; 12: 460-468.

10. Shen Q-D, Zhu H-Y, Wang L et al. Gemcitabine-oxaliplatin plus rituximab (R-GemOx) as first-line treatment in elderly patients with diffuse large B-cell lymphoma: a single-arm, open-label, phase 2 
trial. The Lancet. Haematology 2018; 5: e261-e269.

11. Gini G, Tani M, Tucci A et al. Lenalidomide and Rituximab (ReRi) As Front Line Chemo-Free Therapy of Elderly Frail Patients with Diffuse Large B-Cells Lymphoma. a Phase II Study of the Fondazione Italiana Linfomi (FIL). Blood 2019; 134 (Supplement_1): 2880. .

12. Liu $P$, Han $Y$, Jiang SY et al. A retrospective analysis of real-world outcomes of elderly Chinese patients with diffuse large B-cell lymphoma. Chin Med J (Engl) 2019; 132: 1807-1814.

13. Swerdlow SH, Campo E, Harris NL et al. WHO Classification of Tumours of Haematopoietic and Lymphoid Tissues. International Agency for Research on Cancer (IARC) 69372 Lyon Cedex 08, France 2017.

14. Bertrand Coiffier, Eric Lepage, Josette Briere et al. CHOP chemotherapy plus rituximab compared with CHOP alone in elderly patients with diffuse large-B-cell lymphoma. N Engl J Med 2002; Jan $24 ; 346(4):: 235-242$.

15. Coiffier B, Thieblemont C, Van Den Neste E et al. Long-term outcome of patients in the LNH-98.5 trial, the first randomized study comparing rituximab-CHOP to standard CHOP chemotherapy in DLBCL patients: a study by the Groupe d'Etudes des Lymphomes de l'Adulte. Blood 2010; 116: 2040-2045.

16. He HX, Gao Y, Bai B et al. The beneficial effect of Escalated-R-CHOP-21 for the treatment of diffuse large B-cell lymphoma in elderly male patients: A propensity-matched cohort study. Cancer Med 2021.

17. Pfreundschuh M, Murawski N, Zeynalova S et al. Optimization of rituximab for the treatment of DLBCL: increasing the dose for elderly male patients. British journal of haematology 2017; 179: 410420 .

18. Pfreundschuh M, Poeschel V, Zeynalova S et al. Optimization of rituximab for the treatment of diffuse large B-cell lymphoma (II): extended rituximab exposure time in the SMARTE-R-CHOP-14 trial of the german high-grade non-Hodgkin lymphoma study group. Journal of clinical oncology : official journal of the American Society of Clinical Oncology 2014; 32: 4127-4133.

19. Lugtenburg PJ, de Nully Brown P, van der Holt B et al. Rituximab-CHOP With Early Rituximab Intensification for Diffuse Large B-Cell Lymphoma: A Randomized Phase III Trial of the HOVON and the Nordic Lymphoma Group (HOVON-84). Journal of clinical oncology : official journal of the American Society of Clinical Oncology 2020; 38: 3377-3387.

20. Pfreundschuh M, Schubert J, Ziepert M et al. Six versus eight cycles of bi-weekly CHOP-14 with or without rituximab in elderly patients with aggressive CD20+ B-cell lymphomas: a randomised controlled trial (RICOVER-60). The Lancet. Oncology 2008; 9: 105-116.

21. Delarue R, Tilly H, Mounier N et al. Dose-dense rituximab-CHOP compared with standard rituximabCHOP in elderly patients with diffuse large B-cell lymphoma (the LNH03-6B study): a randomised phase 3 trial. The Lancet Oncology 2013; 14: 525-533.

22. Sehn LH, Congiu AG, Culligan DJ et al. No Added Benefit of Eight Versus Six Cycles of CHOP When Combined with Rituximab in Previously Untreated Diffuse Large B-Cell Lymphoma Patients: Results from the International Phase III GOYA Study. Blood 2018; 132 (Supplement 1): 783. . 2018. 
23. WL\sterlid T, Biccler JL, Brown PN et al. Six cycles of R-CHOP-21 are not inferior to eight cycles for treatment of diffuse large B-cell lymphoma: a Nordic Lymphoma Group Population-based Study. Annals of oncology : official journal of the European Society for Medical Oncology 2018; 29: 18821883.

24. Cunningham D, Hawkes EA, Jack A et al. Rituximab plus cyclophosphamide, doxorubicin, vincristine, and prednisolone in patients with newly diagnosed diffuse large B-cell non-Hodgkin lymphoma: a phase 3 comparison of dose intensification with 14-day versus 21-day cycles. Lancet (London, England) 2013; 381: 1817-1826.

25. Eyre TA, Martinez-Calle N, Hildyard C et al. Impact of intended and relative dose intensity of R-CHOP in a large, consecutive cohort of elderly diffuse large B-cell lymphoma patients treated with curative intent: no difference in cumulative incidence of relapse comparing patients by age. Journal of internal medicine 2019; 285: 681-692.

26. Meguro A, Ozaki K, Sato K et al. Rituximab plus 70\% cyclophosphamide, doxorubicin, vincristine and prednisone for Japanese patients with diffuse large B-cell lymphoma aged 70 years and older. Leuk Lymphoma 2012; 53: 43-49.

27. Scott DW, Wright GW, Williams PM et al. Determining cell-of-origin subtypes of diffuse large B-cell lymphoma using gene expression in formalin-fixed paraffin-embedded tissue. Blood 2014; 123: 12141217.

28. Hans CP, Weisenburger DD, Greiner TC et al. Confirmation of the molecular classification of diffuse large B-cell lymphoma by immunohistochemistry using a tissue microarray. Blood 2004; 103: 275282.

29. Mareschal S, Lanic HIn, Ruminy P et al. The proportion of activated B-cell like subtype among de novo diffuse large B-cell lymphoma increases with age. Haematologica 2011; 96: 1888-1890.

30. Lenz G, Wright G, Dave SS et al. Stromal gene signatures in large-B-cell lymphomas. The New England journal of medicine 2008; 359: 2313-2323.

31. Ruppert AS, Dixon JG, Salles G et al. International prognostic indices in diffuse large B-cell lymphoma: a comparison of IPI, R-IPI, and NCCN-IPI. Blood 2020; 135 (23): 2041-2048. .

32. Spina M, Merli F, Puccini B et al. Definition and Validation of the New Elderly Prognostic Index (EPI) for Elderly Patients with Diffuse Large B-Cell Lymphoma Integrating Geriatric and Clinical Assessment: Results of the Prospective "Elderly Project" on 1353 Patients By the Fondazione Italiana Linfomi. Blood 2019; 134 (Supplement_1): 398. .

33. Merli F, Luminari S, Tucci A et al. Simplified Geriatric Assessment in Older Patients With Diffuse Large B-Cell Lymphoma: The Prospective Elderly Project of the Fondazione Italiana Linfomi. Journal of clinical oncology : official journal of the American Society of Clinical Oncology 2021; 39: 12141222.

34. Hamaker ME, Prins MC, Stauder R. The relevance of a geriatric assessment for elderly patients with a haematological malignancy-a systematic review. Leukemia research 2014; 38: 275-283. 
35. Lin RJ, Behera M, Diefenbach CS, Flowers CR. Role of anthracycline and comprehensive geriatric assessment for elderly patients with diffuse large B-cell lymphoma. Blood 2017; 130: 2180-2185.

\section{Tables}

Table1. Clinical characteristics of 239 Chinese elderly patients (age $\geq 70$ years) with DLBCL at diagnosis. 


\begin{tabular}{|c|c|c|c|c|}
\hline Parameters & $\begin{array}{l}\text { All patients } \\
\mathrm{n}=239\end{array}$ & $\begin{array}{l}70-79 \text { years } \\
n=200\end{array}$ & $\begin{array}{l}\geq 80 \text { years } \\
n=39\end{array}$ & $p$ value \\
\hline Median age & $75.4(70-91)$ & $74.0(70-79)$ & $82.6(80-91)$ & \\
\hline Sex & & & & 0.341 \\
\hline Male & $143(59.8 \%)$ & 117 (58.5\%) & 26 (66.7\%) & \\
\hline Female & $96(40.2 \%)$ & $83(41.5 \%)$ & 13 (33.3\%) & \\
\hline ECOG score & & & & 0.507 \\
\hline $0-1$ & $193(80.8 \%)$ & $163(81.5 \%)$ & 30 (76.9\%) & \\
\hline$\geq 2$ & $46(19.2 \%)$ & 37 (18.5\%) & $9(23.1 \%)$ & \\
\hline B symptoms & & & & 0.527 \\
\hline Yes & $174(72.8 \%)$ & $144(72.0 \%)$ & 30 (76.9\%) & \\
\hline No & $65(27.2 \%)$ & $56(28.0 \%)$ & 9 (23.1\%) & \\
\hline Subtype & & & & 0.759 \\
\hline GCB & $52(21.8 \%)$ & $44(22.0 \%)$ & 8 (20.5\%) & \\
\hline non-GCB & $148(61.9 \%)$ & $122(61.0 \%)$ & $26(66.7 \%)$ & \\
\hline Unknown & 39 (16.3\%) & $34(17.0 \%)$ & $5(12.8 \%)$ & \\
\hline Ki-67 index & & & & 0.784 \\
\hline$>90 \%$ & 7 (2.9\%) & $6(3.0 \%)$ & $1(2.6 \%)$ & \\
\hline$\leq 90 \%$ & $192(80.3 \%)$ & $162(81.0 \%)$ & $30(76.9 \%)$ & \\
\hline Unknown & $40(16.7 \%)$ & $32(16.0 \%)$ & 8 (20.5\%) & \\
\hline Number of extra-nodal involvement & & & & 0.084 \\
\hline 0 or 1 & $174(74.5 \%)$ & $150(75.0 \%)$ & $24(61.5 \%)$ & \\
\hline$\geq 2$ & $65(27.2 \%)$ & $50(25.0 \%)$ & $15(38.5 \%)$ & \\
\hline Bone marrow involvement & & & & 0.442 \\
\hline Yes & $12(5.0 \%)$ & $11(5.5 \%)$ & $1(2.6 \%)$ & \\
\hline No & $227(95 \%)$ & $189(94.5 \%)$ & $38(97.4 \%)$ & \\
\hline Ann Arbor stage & & & & 0.191 \\
\hline$|-I|$ & $96(40.2 \%)$ & $84(42.0 \%)$ & $12(30.8 \%)$ & \\
\hline III-IV & 143 (59.8\%) & 116 (58.0\%) & 27 (69.2\%) & \\
\hline
\end{tabular}




\begin{tabular}{|lllll|}
\hline Bulky disease & $38(15.9)$ & $33(16.5 \%)$ & $5(12.8 \%)$ & 0.565 \\
\hline Yes & $201(84.1 \%)$ & $167(83.5 \%)$ & $34(87.2 \%)$ & \\
\hline Serum LDH & & & & 0.900 \\
\hline Normal & $102(42.7 \%)$ & $85(42.5 \%)$ & $17(43.6 \%)$ & \\
\hline Elevated & $137(57.3 \%)$ & $115(57.5 \%)$ & $22(56.4 \%)$ & 0.299 \\
IPI score & & & & \\
\hline 1 & $52(21.8 \%)$ & $44(22.0 \%)$ & $8(20.5 \%)$ & \\
\hline 2 & $61(25.5 \%)$ & $55(27.5 \%)$ & $6(15.4 \%)$ & \\
\hline 3 & $65(27.2 \%)$ & $54(27.0 \%)$ & $11(28.2 \%)$ & \\
\hline $4-5$ & $61(25.5 \%)$ & $47(23.5 \%)$ & $14(35.9 \%)$ & \\
\hline Comorbidities & & & & \\
\hline Yes & $205(85.8 \%)$ & $171(85.5 \%)$ & $34(87.2 \%)$ & \\
\hline No & $34(14.2 \%)$ & $29(14.5 \%)$ & $5(12.8 \%)$ & \\
\hline
\end{tabular}

ECOG: eastern cooperative oncology group. GCB: germinal center B-cell like. LDH: lactate dehydrogenase. IPI: international prognostic index.

Table2. Initial treatment regimens of Chinese elderly patients with DLBCL $(n=239)$. 


\begin{tabular}{|c|c|c|c|c|}
\hline Parameters & $\begin{array}{l}\text { All patients } \\
n=239\end{array}$ & $\begin{array}{l}\text { 70-79 years } \\
n=200\end{array}$ & $\begin{array}{l}\geq 80 \text { years } \\
n=39\end{array}$ & $p$ value \\
\hline Therapy options & & & & 0.794 \\
\hline Chemotherapy & $154(64.4 \%)$ & $128(64.0 \%)$ & $26(66.7 \%)$ & \\
\hline Radiotherapy & $2(0.8 \%)$ & $1(0.5 \%)$ & $1(2.6 \%)$ & \\
\hline Surgery & $4(1.7 \%)$ & $3(1.5 \%)$ & $1(2.6 \%)$ & \\
\hline $\begin{array}{l}\text { Chemotherapy } \\
\text { +Radiotherapy }\end{array}$ & $27(11.3 \%)$ & $24(12.0 \%)$ & $3(7.7 \%)$ & \\
\hline $\begin{array}{l}\text { Surgery } \\
+ \text { Chemotherapy }\end{array}$ & $29(12.1 \%)$ & $25(12.5 \%)$ & $4(10.3 \%)$ & \\
\hline $\begin{array}{l}\text { Surgery } \\
\text { +Radiotherapy }\end{array}$ & $3(1.3 \%)$ & $2(1.0 \%)$ & $1(2.6 \%)$ & \\
\hline $\begin{array}{l}\text { Surgery } \\
\text { +Chemotherapy } \\
\text { +Radiotherapy }\end{array}$ & $9(3.8 \%)$ & $7(3.5 \%)$ & $2(5.1 \%)$ & \\
\hline Untreated & $11(4.6 \%)$ & $10(5.0 \%)$ & $1(2.6 \%)$ & \\
\hline Rituximab & & & & 0.350 \\
\hline Yes & $156(65.3 \%)$ & $128(64.0 \%)$ & $28(71.8 \%)$ & \\
\hline No & $83(34.7 \%)$ & $72(36.0 \%)$ & $11(28.2 \%)$ & \\
\hline Dose reduction of chemotherapy & & & & $<0.001$ \\
\hline Yes & $53(22.2 \%)$ & $37(18.5 \%)$ & $16(41.0 \%)$ & \\
\hline No & $147(61.5 \%)$ & $135(67.5 \%)$ & $12(30.8 \%)$ & \\
\hline Unknown & 39 (16.3\%) & 28 (14.0\%) & $11(28.2 \%)$ & \\
\hline
\end{tabular}

Table3. Chemotherapy of Chinese elderly patients with DLBCL $(n=219)$. 


\begin{tabular}{|c|c|c|c|c|}
\hline Parameters & $\begin{array}{l}\text { All patients } \\
\mathrm{n}=\mathbf{2 1 9}\end{array}$ & $\begin{array}{l}70-79 \text { years } \\
n=184\end{array}$ & $\begin{array}{l}\geq 80 \text { years } \\
n=35\end{array}$ & $p$ value \\
\hline Rituximab & & & & 0.211 \\
\hline Yes & $156(71.2 \%)$ & $128(69.6 \%)$ & $28(80.0 \%)$ & \\
\hline No & $63(28.8 \%)$ & $56(30.4 \%)$ & $7(20.0 \%)$ & \\
\hline Regimens & & & & 0.004 \\
\hline $\mathrm{CHOP}$ & $185(84.5 \%)$ & $162(88.0 \%)$ & $23(65.7 \%)$ & \\
\hline CVP & $15(6.8 \%)$ & $10(5.4 \%)$ & $5(14.3 \%)$ & \\
\hline Others & $19(8.7 \%)$ & $12(6.5 \%)$ & $7(20.0 \%)$ & \\
\hline Chemotherapy dose reduction & & & & $<0.001$ \\
\hline Yes & $53(24.2 \%)$ & $37(20.1 \%)$ & $16(45.7 \%)$ & \\
\hline No & $147(67.1 \%)$ & $135(73.4 \%)$ & $12(34.3 \%)$ & \\
\hline Unknown & $19(8.7 \%)$ & $12(6.5 \%)$ & $7(20.0 \%)$ & \\
\hline Therapy cycles & & & & 0.740 \\
\hline$<6$ & $112(51.1 \%)$ & $95(51.6 \%)$ & $17(48.6 \%)$ & \\
\hline$\geq 6$ & $107(48.9 \%)$ & $89(48.4 \%)$ & $18(51.4 \%)$ & \\
\hline Prophylaxis with G-CSF & & & & 0.060 \\
\hline Yes & $44(20.1 \%)$ & $32(17.4 \%)$ & $22(62.9 \%)$ & \\
\hline No & $161(73.5 \%)$ & $139(75.5 \%)$ & $12(34.2 \%)$ & \\
\hline Unknown & $14(6.4 \%)$ & 13 (7.1\%) & $1(2.9 \%)$ & \\
\hline
\end{tabular}

G-CSF: granulocyte colony-stimulating factor.

Table4A. Multivariate analysis of PFS in 239 Chinese elderly patients with DLBCL. 


\begin{tabular}{|lllllll|}
\hline Parameters & $\boldsymbol{\beta}$ & SE & Wald & HR & $95 \%$ Cl & $\boldsymbol{p}$ value \\
\hline Stage III/IV & 0.495 & 0.237 & 4.358 & 1.640 & $1.031-2.610$ & $\mathbf{0 . 0 3 7}$ \\
\hline ECOG score $\geq 2$ & 0.232 & 0.258 & 0.807 & 1.261 & $0.760-2.092$ & 0.369 \\
\hline Serum LDH >normal & 0.073 & 0.213 & 0.117 & 1.076 & $0.708-1.634$ & 0.733 \\
\hline Extranodal involvement $>1$ site & -0.020 & 0.236 & 0.008 & 0.980 & $0.616-1.557$ & 0.931 \\
\hline B symptoms & 0.144 & 0.218 & 0.440 & 1.155 & $0.754-1.770$ & 0.507 \\
\hline Bone marrow involvement & 0.129 & 0.455 & 0.080 & 1.137 & $0.466-2.774$ & 0.777 \\
\hline Rituximab & -0.475 & 0.213 & 4.984 & 0.622 & $0.410-0.944$ & $\mathbf{0 . 0 2 6}$ \\
\hline
\end{tabular}

Table4B. Multivariate analysis of OS in 239 Chinese elderly patients with DLBCL.

\begin{tabular}{|lllllll|}
\hline Parameters & $\boldsymbol{\beta}$ & SE & Wald & HR & $95 \%$ Cl & p value \\
\hline Stage III/IV & 0.137 & 0.294 & 0.215 & 1.146 & $0.644-2.041$ & 0.643 \\
\hline ECOG score $\geq 2$ & 0.121 & 0.337 & 0.129 & 1.129 & $0.583-2.188$ & 0.719 \\
\hline Serum LDH >normal & -0.063 & 0.267 & 0.056 & 0.939 & $0.556-1.585$ & 0.813 \\
\hline Extralnodal involvement $>1$ site & 0.236 & 0.291 & 0.656 & 1.266 & $0.716-2.237$ & 0.418 \\
\hline B symptoms & 0.364 & 0.274 & 1.771 & 1.440 & $0.842-2.463$ & 0.183 \\
\hline Bone marrow involvement & 0.243 & 0.640 & 0.144 & 1.275 & $0.363-4.473$ & 0.704 \\
\hline Dose reduction & 0.602 & 0.279 & 4.650 & 1.825 & $1.056-3.154$ & $\mathbf{0 . 0 3 1}$ \\
\hline Rituximab & -0.787 & 0.278 & 8.016 & 0.455 & $0.264-0.785$ & $\mathbf{0 . 0 0 5}$ \\
\hline
\end{tabular}

Table5. Summary of adverse events $(n=187)$. 


\begin{tabular}{|c|c|c|c|}
\hline Parameters & $\begin{array}{l}\text { All patients } \\
\mathrm{n}=187\end{array}$ & $\begin{array}{l}\text { 70-79 years } \\
n=158\end{array}$ & $\begin{array}{l}\geq 80 \text { years } \\
\mathrm{n}=29\end{array}$ \\
\hline Anemia & $146(78.1 \%)$ & $126(79.7 \%)$ & $20(69.0 \%)$ \\
\hline Leukopenia & 149 (79.7\%) & $127(80.4 \%)$ & $22(75.9 \%)$ \\
\hline Neutropenia & $128(68.4 \%)$ & $109(69.0 \%)$ & $19(65.5 \%)$ \\
\hline Febrile neutropenia & $26(13.9 \%)$ & $20(12.7 \%)$ & $6(20.7 \%)$ \\
\hline Thrombocytopenia & $59(31.6 \%)$ & $55(34.8 \%)$ & $4(13.8 \%)$ \\
\hline Thrombus & $9(4.8 \%)$ & $8(5.1 \%)$ & $1(3.4 \%)$ \\
\hline Fatigue & $111(59.4 \%)$ & $85(53.8 \%)$ & $26(89.7 \%)$ \\
\hline Nausea/vomiting & $148(79.1 \%)$ & $122(77.2 \%)$ & $26(89.7 \%)$ \\
\hline Hypo-albumin & $128(68.4 \%)$ & $101(63.9 \%)$ & $27(93.1 \%)$ \\
\hline $\begin{array}{l}\text { Aspartate aminotransferase } \\
\text { increased }\end{array}$ & $59(31.0 \%)$ & $51(32.3 \%)$ & $8(27.7 \%)$ \\
\hline Elevated creatinine & $13(5.9 \%)$ & $10(6.3 \%)$ & $1(3.4 \%)$ \\
\hline Neuro-toxicities & $6(3.2 \%)$ & $6(3.8 \%)$ & 0 \\
\hline Cardio-toxicities & $11(5.9 \%)$ & $10(6.3 \%)$ & $1(3.4 \%)$ \\
\hline Death during treatment & $28(15.0 \%)$ & $20(12.7 \%)$ & $8(27.6 \%)$ \\
\hline
\end{tabular}

Table6. Summary of $\geq$ grade 3 hematological adverse events $(n=187)$.

\begin{tabular}{|llll|}
\hline Parameters & $\begin{array}{l}\text { All patients } \\
\mathbf{n = 1 8 7}\end{array}$ & $\begin{array}{l}\mathbf{7 0 - 7 9} \text { years } \\
\mathbf{n = 1 5 8}\end{array}$ & $\begin{array}{l}\mathbf{2 8 0} \text { years } \\
\mathbf{n}=\mathbf{2 9}\end{array}$ \\
\hline Anemia & $23(12.3 \%)$ & $22(13.9 \%)$ & $1(3.4 \%)$ \\
\hline Leukopenia & $76(40.6 \%)$ & $62(39.2 \%)$ & $14(48.3 \%)$ \\
\hline Neutropenia & $71(38.0 \%)$ & $57(36.1 \%)$ & $14(48.3 \%)$ \\
\hline Febrile neutropenia & $26(13.9 \%)$ & $20(12.7 \%)$ & $6(20.7 \%)$ \\
\hline Thrombocytopenia & $15(8.0 \%)$ & $14(8.9 \%)$ & $1(3.4 \%)$ \\
\hline Total & $94(50.3 \%)$ & $78(49.4 \%)$ & $16(55.2 \%)$ \\
\hline
\end{tabular}


Figures
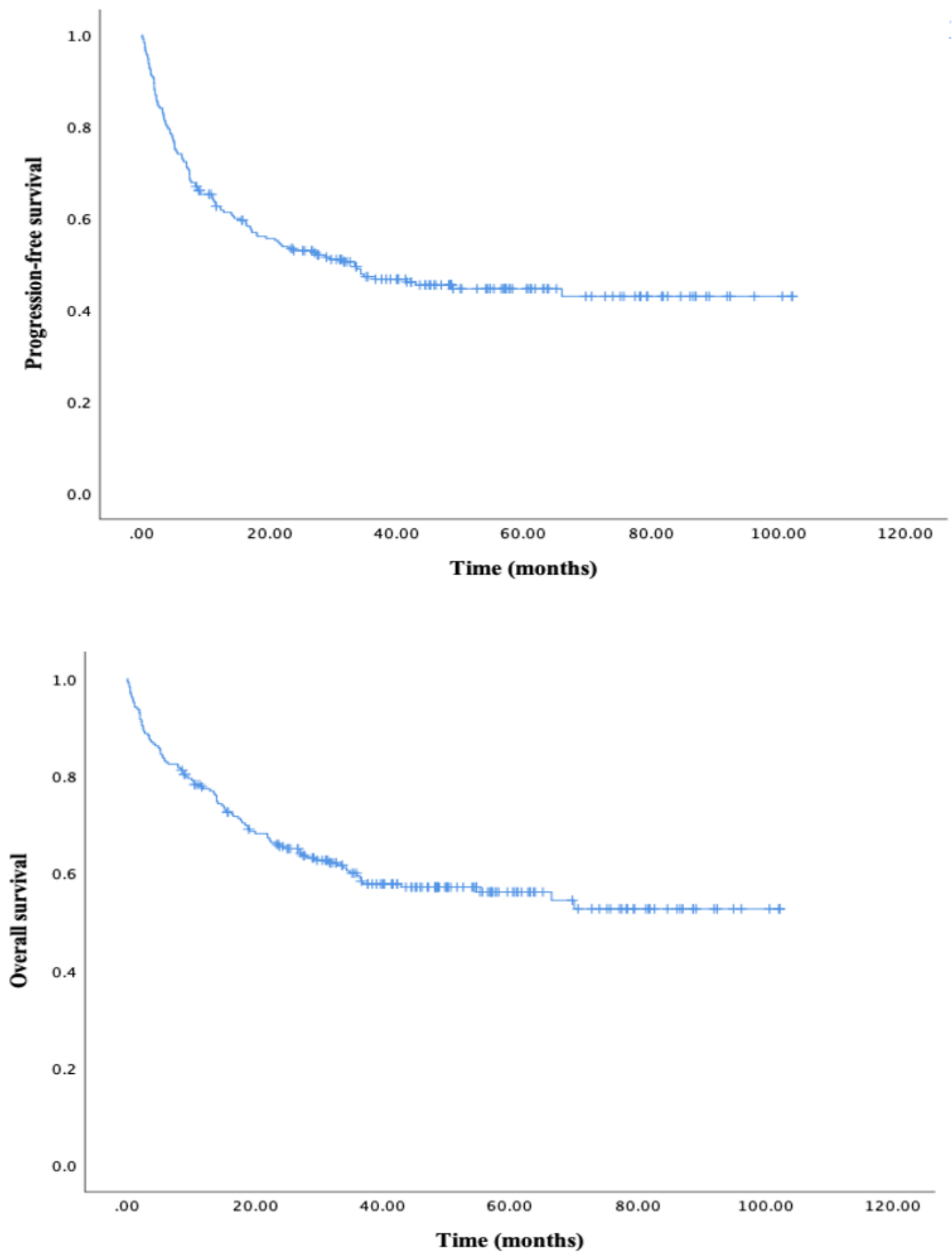

\section{Figure 1}

(n=239). Kaplan-Meier survival curve of progression-free survival and overall survival of 239 DLBCL patients. The 2-y PFS and 2-y OS were 53.0\% (95\% Cl: 46.5-59.5) and 65.5\% (95\% Cl: 59.4-71.2), the 5- 
year PFS and OS were 44.7\% (95\% Cl: 38.0-51.4), 56.1\% (95\% Cl: 49.4-62.8), and the median PFS and media OS were 42.1 months and 96.4 months.
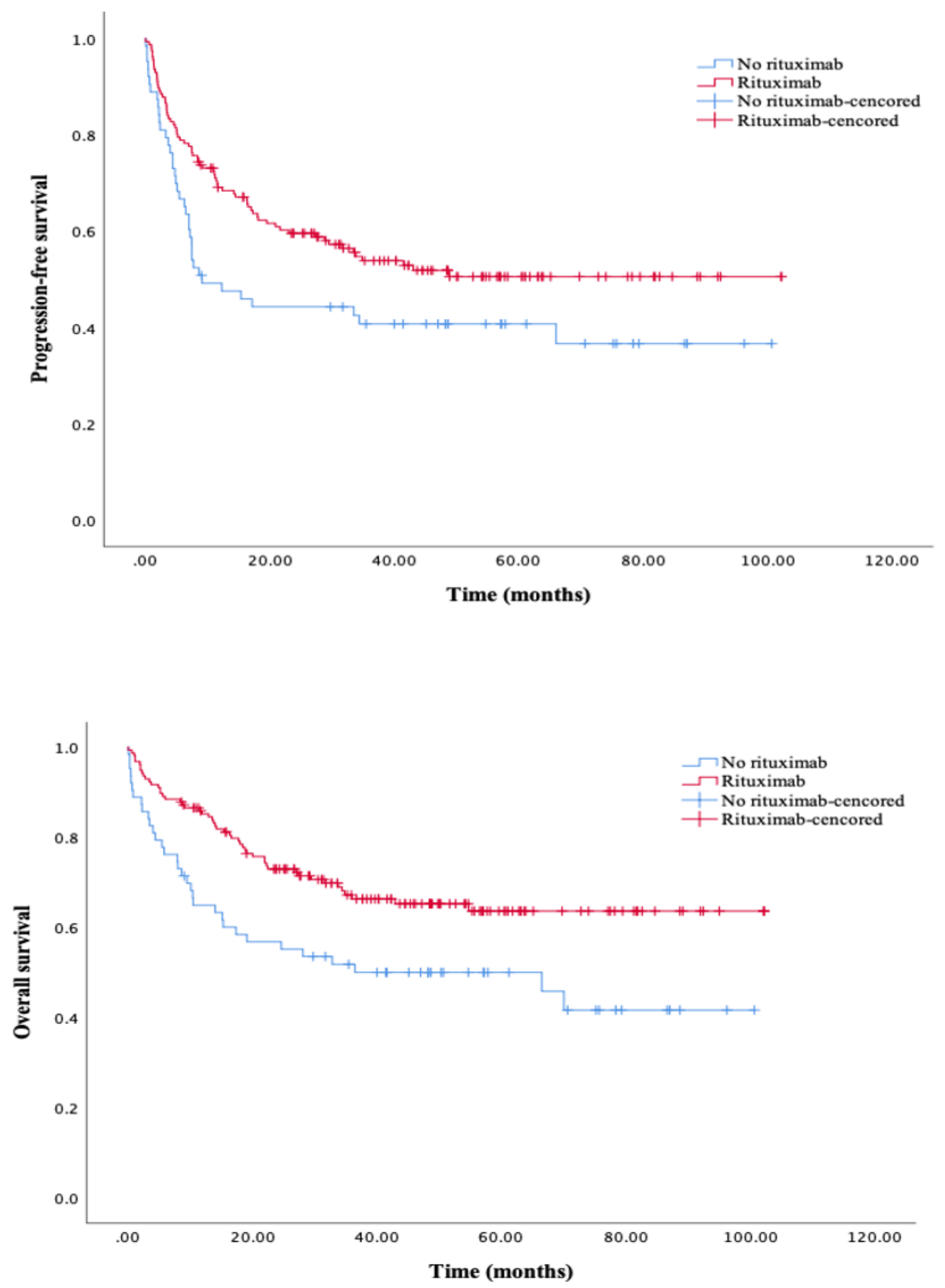

Figure 2

(n=219). Kaplan-Meier survival curve of PFS and OS for elderly patients with DLBCL stratified by rituximab usage. The 2-y PFS rate in rituximab-containing treatment group and without rituximab group 
was $59.5 \%, 44.2 \%, p 0.031$; the $2-y$ OS rate in rituximab-containing treatment group and without rituximab group was $72.9 \%, 56.8 \%, p 0.008$.
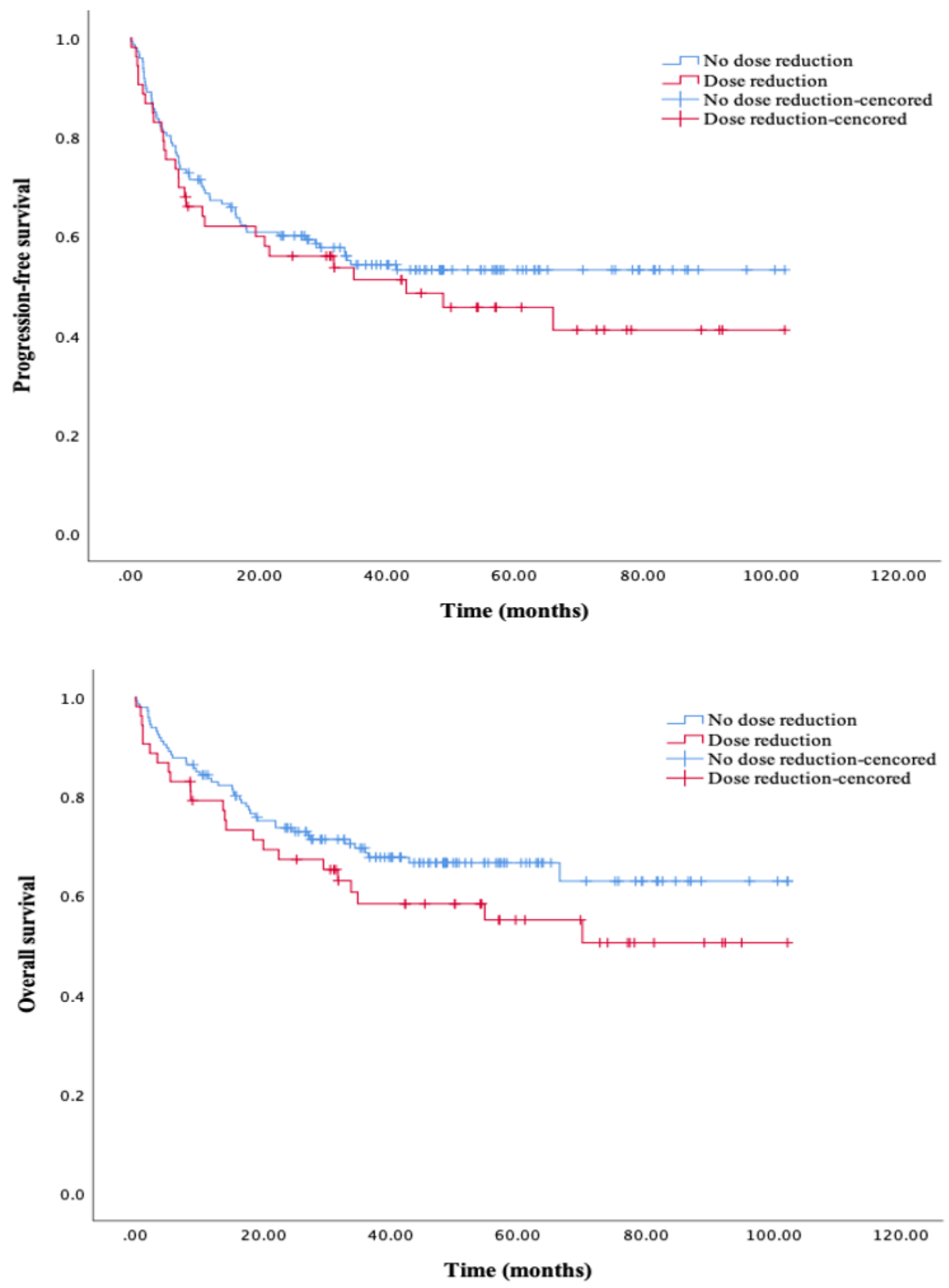

Figure 3 
( $\mathbf{n = 2 0 0 ) . ~ K a p l a n - M e i e r ~ s u r v i v a l ~ c u r v e ~ o f ~ P F S ~ a n d ~ O S ~ f o r ~ e l d e r l y ~ p a t i e n t s ~ w i t h ~ D L B C L ~ s t r a t i f i e d ~ b y ~ r e d u c e d ~}$ chemotherapy dose. The 2-y PFS rate in group of reduced chemotherapy dose and in group of standard dose was $56.0 \%, 60.1 \%, p 0.384$, the 2 -y OS rate in group of reduced chemotherapy dose and in group of standard dose was $67.3 \%, 73.7 \%, p 0.139$.
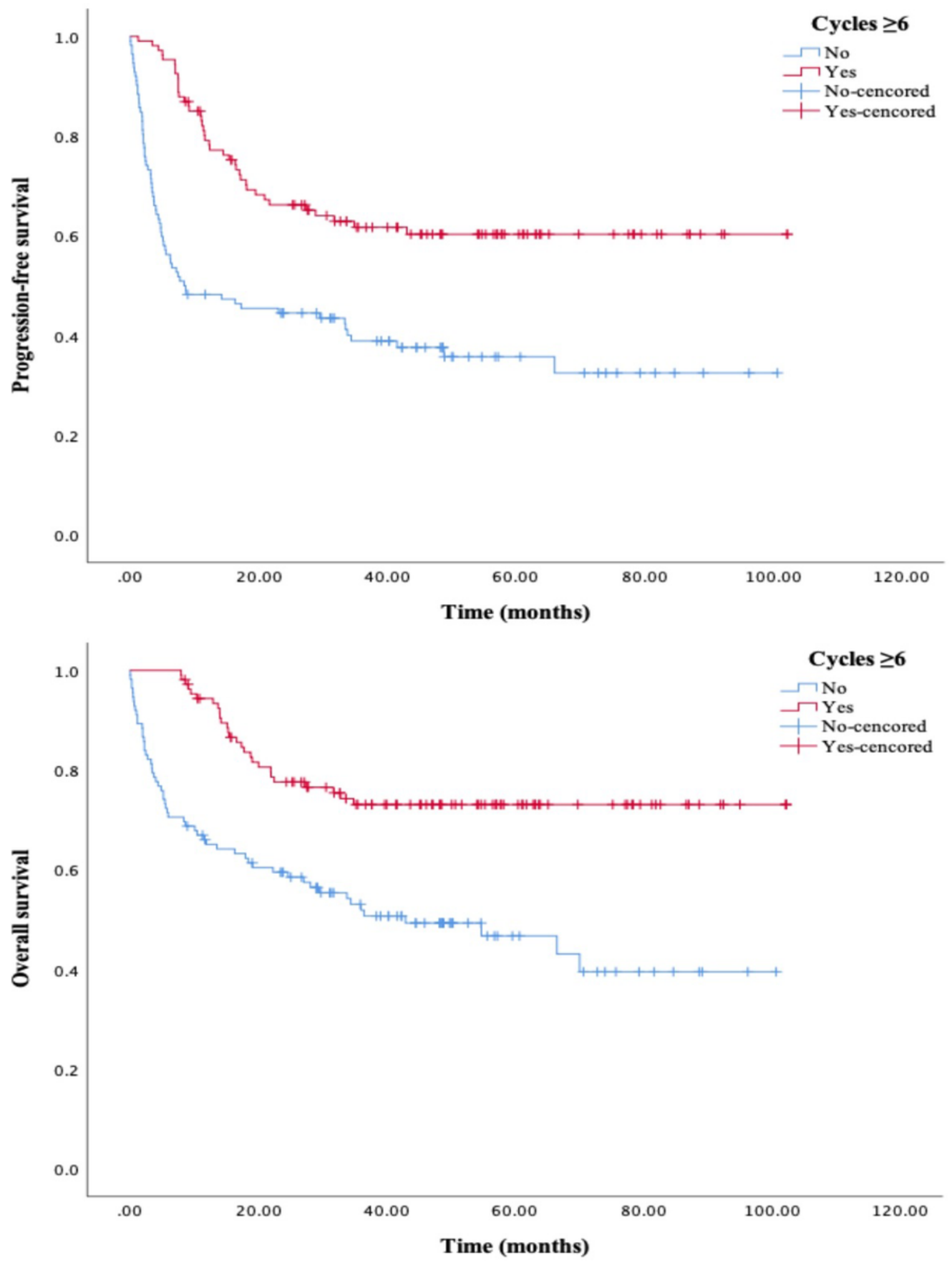
Figure 4

( $\mathbf{n = 2 1 9 ) . ~ K a p l a n - M e i e r ~ s u r v i v a l ~ c u r v e ~ o f ~ P F S ~ a n d ~ O S ~ f o r ~ e l d e r l y ~ p a t i e n t s ~ w i t h ~ D L B C L ~ s t r a t i f i e d ~ b y ~}$ chemotherapy cycles. The 2-y PFS rate in group of $<6$ chemotherapy cycles and in group of $\geq 6$ cycles was $44.5 \%, 66.2 \%, p 0.000$, the 2 -y OS rate in group of in group of $<6$ chemotherapy cycles and in group of $\geq 6$ cycles was $59.5 \%, 77.6 \%, p 0.000$.

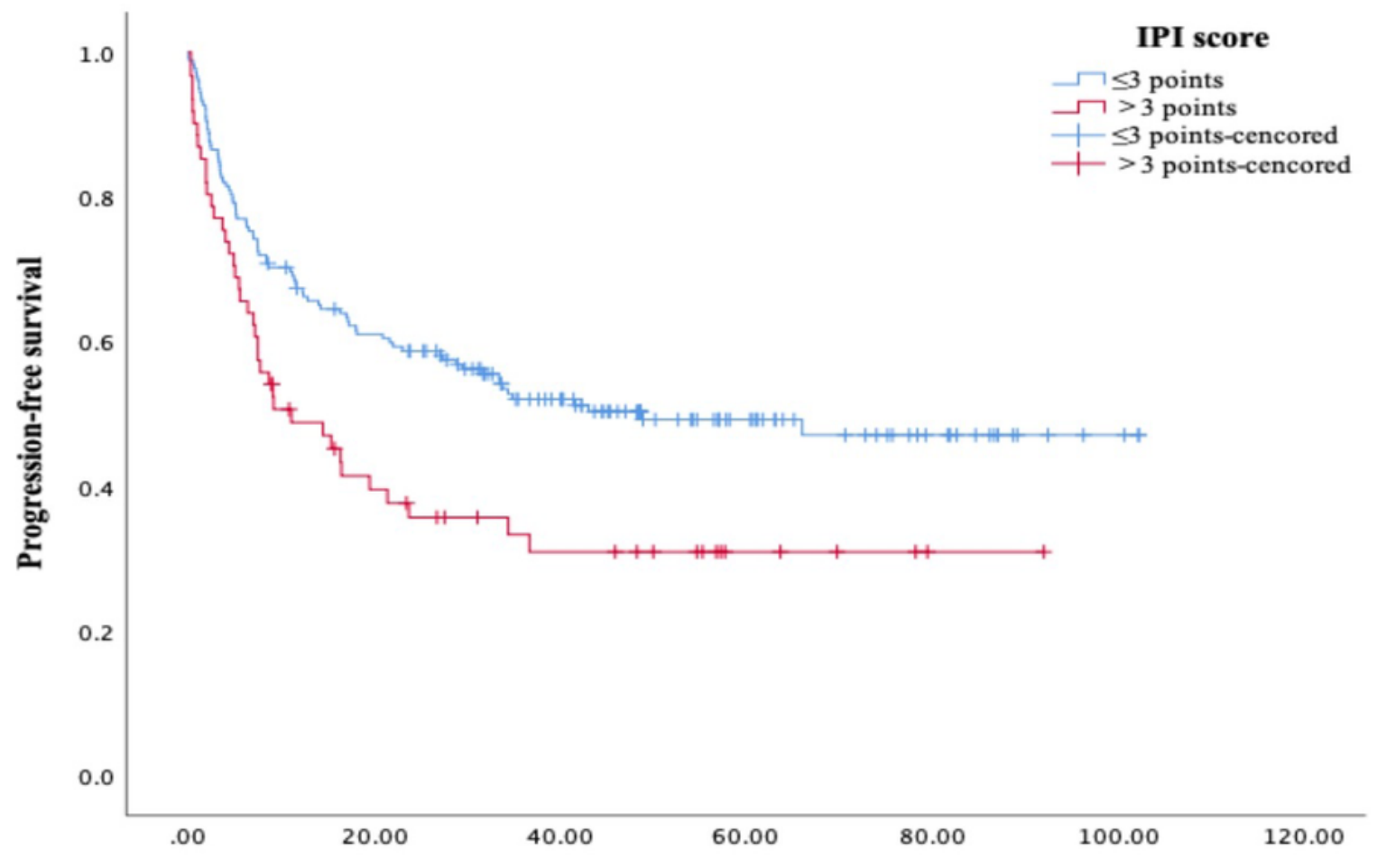

Time (months)

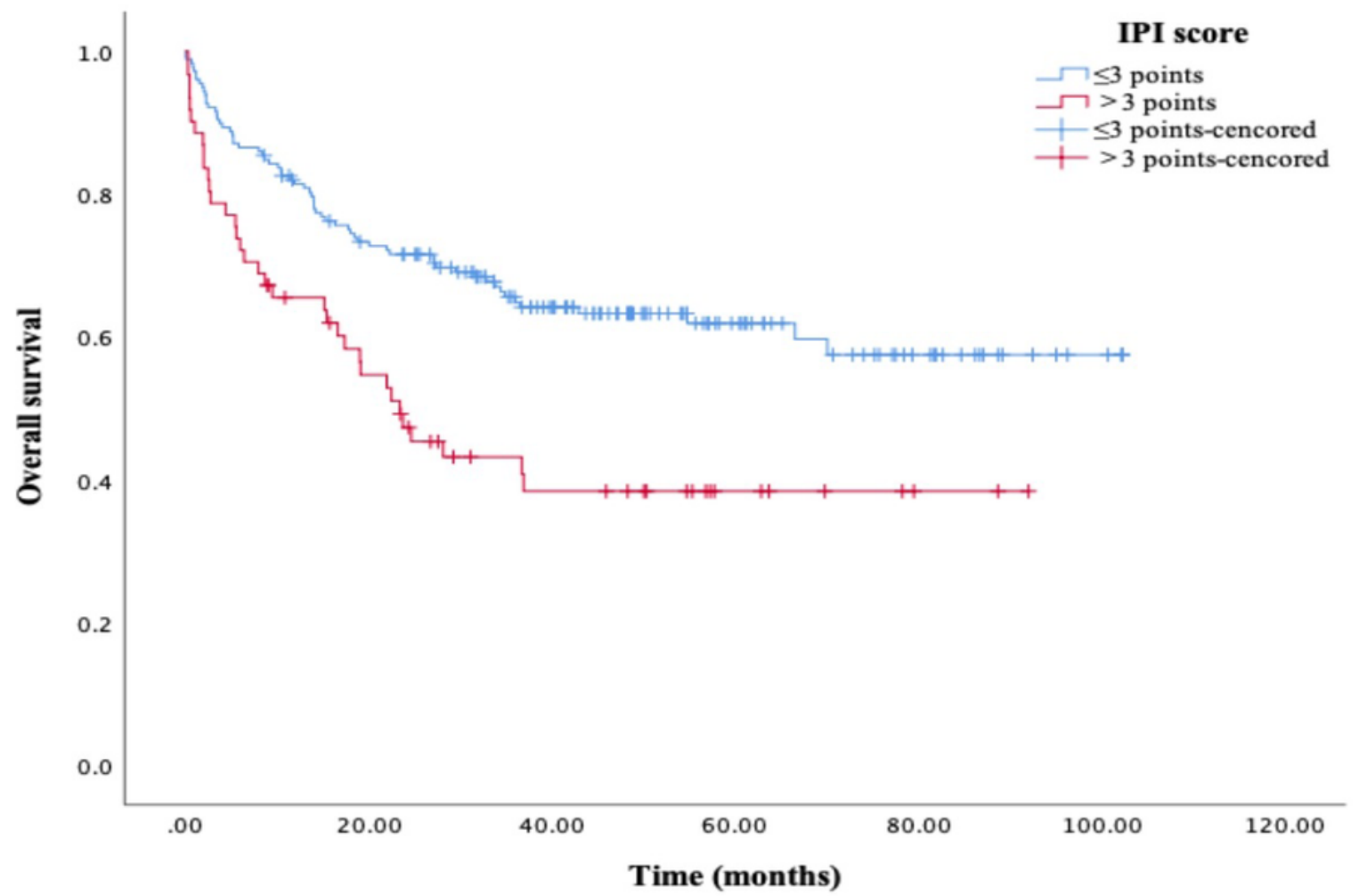




\section{Figure 5}

( $\mathbf{n = 2 3 9 ) .}$ Kaplan-Meier survival curve of PFS and OS for elderly patients with DLBCL stratified by IPI score. The 2-y PFS rate in group of IPI $\leq 3$ and of IPI $>3$ was $58.7 \%, 35.7 \%, p 0.005$, the 2-y OS rate in group of IPI $\leq 3$ and of $\mathrm{IPI}>3$ was $71.5 \%, 47.3 \%, p 0.001$.

\section{Supplementary Files}

This is a list of supplementary files associated with this preprint. Click to download.

- supplementaryfigures.docx

- supplementarytables.docx 